\title{
Germanica
}

\section{De la vieillesse et des fins ultimes. Réflexions sur la vieillesse dans l'œuvre de Gabriele Wohmann}

Über das Alter und die letzten Fragen. Reflexionen über das Alter im Werk Gabriele Wohmanns.

On aging and the final questions. Reflections about aging in Gabriele Wohmann's work.

\section{Benoît Pivert}

\section{OpenEdition Journals}

Édition électronique

URL : http://journals.openedition.org/germanica/1569

DOI : 10.4000/germanica.1569

ISSN : 2107-0784

\section{Éditeur}

Université de Lille

\section{Édition imprimée}

Date de publication : 10 juillet 2012

Pagination : 61-78

ISBN : 9782913857292

ISSN : 0984-2632

\section{Référence électronique}

Benoît Pivert, « De la vieillesse et des fins ultimes. Réflexions sur la vieillesse dans l'œuvre de Gabriele Wohmann », Germanica [En ligne], 50 | 2012, mis en ligne le 19 juillet 2012, consulté le 06 octobre 2020. URL : http://journals.openedition.org/germanica/1569; DOI : https://doi.org/10.4000/ germanica. 1569

Ce document a été généré automatiquement le 6 octobre 2020.

(c) Tous droits réservés 


\title{
De la vieillesse et des fins ultimes. Réflexions sur la vieillesse dans l'œuvre de Gabriele Wohmann
}

\author{
Über das Alter und die letzten Fragen. Reflexionen über das Alter im Werk \\ Gabriele Wohmanns. \\ On aging and the final questions. Reflections about aging in Gabriele Wohmann's \\ work.
}

\section{Benoît Pivert}

Dans La Vie à l'épreuve du temps, la psychanalyste Catherine Bergeret-Amselek note : « Évoquer la vieillesse en l'appelant par son nom, évoquer la mort ne sont pas des sujets qui font vendre. Aujourd'hui, il faut des propos qui renforcent le déni collectif adopté par une société narcissique qui fait comme si la vieillesse n'existait pas, celle-ci évoquant une décrépitude et une mort qui font horreur $»^{\prime}$. S'il est une œuvre littéraire à laquelle cette citation ne s'applique pas, c'est bien celle de Gabriele Wohmann. Née en 1932 à Darmstadt, la romancière et nouvelliste Gabriele Wohmann - qui n'a toujours pas posé la plume - fêtera en 2012 son quatre-vingtième anniversaire. C'est toutefois bien avant d'atteindre cet âge respectable que l'écrivain s'est penché sur la question de la vieillesse. En 1985, alors qu'elle n'a encore que 53 ans, Gabriele Wohmann publie, avec un mélange de dérision et d'autodérision, Der siebzigste Geburtstag, récit dans lequel elle imagine par avance les "réjouissances » qui l'attendent. Depuis, les personnages âgés sont devenus si nombreux que la vieillesse se dessine comme un leitmotiv dans les œuvres des dernières décennies. C'est même un roman entier à tonalité tchekhovienne que Gabriele Wohmann a consacré au grand âge, Bitte nicht sterben, publié en 1993. De romans en nouvelles, Gabriele Wohmann a dépeint de nombreux "tableaux de genre ", consacrés à la vieillesse dans les dernières décennies $\mathrm{du}$ xxe siècle et la première décennie $\mathrm{du}$ xxie siècle. Son œuvre, quoique sans prétention scientifique, a valeur d'étude gérontologique puisque l'on y retrouve aussi bien la physiologie que la sociologie ou la psychologie du vieillissement. Nous nous attacherons donc dans cette étude à la représentation de ces divers aspects. Mais 
l'œuvre de Gabriele Wohmann va au-delà de la gérontologie. Au fil des années et à mesure que les personnages vieillissaient en même temps que leur auteur, une interrogation s'est faite plus pressante, interrogation métaphysique et réflexion sur la fin de vie et les questions ultimes. C'est ce questionnement qui figure au centre, par exemple, de l'ouvrage Erzählen Sie mir was vom Jenseits (1994) ou plus récemment Sterben ist Mist, der Tod aber schön (2011). Dans une littérature contemporaine dont Dieu est souvent le grand absent, cela constitue indéniablement l'originalité de Gabriele Wohmann et c'est cette voix originale que nous tenterons de faire entendre ici.

\section{Physiologie de la vieillesse}

Disons d'emblée qu'il ne faut pas compter sur G. Wohmann pour trancher l'épineux débat sur la question de l'âge à partir duquel commence la vieillesse. G. Wohmann a compris que «la vieillesse ne se laissait pas appréhender, fixer " ${ }^{2}$, elle préfère donc partir de la représentation du vieillissement « qui est de l'ordre de l'apparence, du biologique, du constatable $»^{3}$. Elle n'ignore pas non plus qu'il existe souvent un décalage entre la façon dont le sujet se perçoit et la réalité que lui renvoie le miroir. Il est certes possible de duper autrui par des artifices cosmétiques mais pour le sujet luimême, il est devenu impossible d'ignorer que le vieillissement a commencé. Cette épreuve du miroir impitoyable est fréquente en littérature. Dans La Vieillesse ${ }^{4}$, Simone de Beauvoir raconte être restée incrédule quand, plantée devant un miroir, elle s'est dit qu'elle avait quarante ans. Ce n'était pourtant que le prodrome des outrages à venir. Une scène similaire se retrouve chez Gabriele Wohmann, venant donner raison à Goethe lorsque ce dernier affirme que l'âge s'empare de nous par surprise. L'héroïne de Der beste Freund ist in dem Himmel (1983) constate :

Pouh, je ressemble vraiment à une vieille femme, avait-elle soupiré intérieurement lorsqu'elle s'était vue par surprise, de mauvaise humeur, dans un miroir. Ce n'est que plus tard qu'elle avait été frappée du fait qu'elle était bel et bien une vieille femme, mais à ce moment-là, comme elle n'avait pu s'empêcher de rire, cette impression d'être une vieille femme s'était déjà dissipée ${ }^{5}$.

Il n'est pas toujours aussi facile de ruser avec soi-même. Dans la nouvelle Verschluck dich nicht (1992), Aurelia Beilstein, gérontologue d'un certain âge, cherche à se rajeunir pour séduire mais il lui faut bien se rendre à la réalité :

La vieillesse? Autrefois, je comprenais immédiatement la prose de Schopenhauer. Autrefois, je pouvais monter une côte sans être essoufflée. Autrefois, le moindre courant d'air ne me perturbait pas et, les cheveux en désordre, j'avais fière allure. Autrefois, je retenais ce que je lisais. Autrefois, je n'avais pas besoin de bêtabloquant ni de lunettes. Autrefois, je songeais à la mort avec jouissance et en toute tranquillité et surtout je ne songeais jamais à ma digestion ${ }^{6}$.

D'aucuns qualifieront de manque de goût cette évocation des fonctions digestives devenues capricieuses mais il faut savoir que Gabriele Wohmann est un écrivain qui n'aime guère les fioritures. Pour elle, comme pour le Général de Gaulle, la vieillesse est un naufrage. Gabriele Wohmann s'inscrit ainsi dans une tradition de représentation littéraire des vicissitudes de l'âge qui remonte au philosophe et poète égyptien Ptah Hotep en 2500 avant J.-C., tradition qui parcourt toute la littérature occidentale et dont on trouve, pour le xxe siècle, un inventaire établi par Béatrice Jongy dans $D^{\prime}$ âge en $\hat{a} g e^{7}$, à partir des témoignages autobiographiques de Tolstoï, Cioran, Léautaud, Gide, 
Cocteau et Ionesco. On se reportera aussi avec profit à l'étude de Claudine Chevallier, "La vieillesse en littérature " ${ }^{8}$. Ces textes sont pour la plupart très sombres. Pour ces écrivains, « la vieillesse est un mal, une infirmité, un âge triste et morbide qui prépare à la mort, [...] la vieillesse est, d'une certaine façon toujours envisagée comme une maladie $"$. Pour G. Wohmann aussi, la vieillesse est avant tout terrible. Rien ne lui insupporte tant que les visions mensongères colportées auprès du grand public, « tous ces seniors alertes avec leurs voyages à prix cassés, qui passent l'hiver à Majorque, [...] rien que de l'hypocrisie. On parle moins volontiers des malades, des impotents, des vieillards atteints de démence sénile dans leurs chambres à deux ou trois lits dans des hospices $\aleph^{10}$. Tout aussi insupportable est cette malhonnêteté qui consiste à faire croire aux personnes âgées qu'un peu de peinture, de nourriture bio et de yoga suffiront à transformer la vieillesse en " âge d'or ». G. Wohmann, elle, se refuse aux enjolivements coutumiers. Elle préfère parler de la calamité d'une vieillesse scélérate. Si son attention se porte tant sur la physiologie, c'est que G. Wohmann a toujours entretenu un rapport particulier au corps, rapport qualifié par Klaus Wellner dans une étude consacrée à l'écrivain d' "hostilité au corps $»^{11}$. La vieillesse, qui passe par le corps, est perçue comme d'autant plus dérangeante. Le tableau qu'en dresse G. Wohmann est toutefois nuancé selon qu'elle parle de l'âge ou du grand âge. Aux moins âgés les moindres maux. La vue décline, comme chez Aniela, l'héroïne de Aus der Nähe gesehen (1992), perdue sans ses lunettes de lecture oubliées à la maison ; la voix devient éraillée comme chez le personnage principal de Der beste Freund ist in dem Himmel (1983), qui avait jadis une voix de soprano ; le corps s'empâte comme celui de Silja Knecht qui, dans Die Bagatelle-Balance (1983), ne voit plus bien pour qui ou pour quoi elle devrait contrôler son poids. Le tableau est tout autre dans le roman fortement autobiographique Bitte nicht sterben dont les protagonistes sont trois vieilles femmes, Louisa, quatre-vingt-onze ans, mère de la narratrice, entourée de ses deux sœurs Marie-Rosa et Bertine. Seule Bertine est légèrement plus jeune. Ces trois vieilles femmes sont observées semaine après semaine par la narratrice et son mari, doubles fictionnels de Gabriele et Reiner Wohmann. Gabriele Wohmann semble tiraillée dans ce roman entre une forme de pudeur pour des personnages que l'on devine être sa mère et ses tantes et la volonté de montrer à quel point le grand âge coïncide avec les derniers outrages :

Il nous faut lutter contre une forme de pruderie à l'égard des faits. Devant l'avenir, nous mettons bas les armes. Nous nous sentons déloyaux, nous avons le sentiment de manquer de tact quand nous faisons ne serait-ce qu'une allusion à l'impotence de la vieillesse, nous nous en tenons à cette expression démodée «ce sont des choses dont on ne parle pas ", avant de retomber dans notre ton badin ${ }^{12}$.

Pourtant la narratrice ne passe pas grand-chose sous silence, ni la cataracte ni les lavements ni encore l'incontinence qui oblige à acheter des couches et contraint les enfants à nettoyer leurs parents comme eux les ont nettoyés lorsqu'ils étaient enfants. Et la narratrice confesse qu'il est bien dur de n'y voir que justice.Comment ose-t-on parler d'un bel âge quand une vieille femme ne peut qu'avoir peur en se levant du canapé, parce qu'elle ne tient plus solidement sur ses jambes, qu'elle a des vertiges, qu'elle ne peut presque plus rien faire, qu'elle ne fait plus que passer le temps sur son canapé ? La mère de la narratrice, incapable de se relever, a déjà passé une nuit dans sa baignoire. Ce délabrement est un fardeau psychologique pour la personne âgée, mais c'est aussi un fardeau pour l'entourage. À chaque nouvelle visite, une question tenaille la narratrice du roman: comment allons-nous les trouver? G. Wohmann décrit 
l'anxiété diffuse qui accompagne les dérèglements physiologiques des êtres chers. Et si les maux d'estomac soudains n'étaient que les prémices du grand final ? C'est cela qui donne à ce roman une tonalité toute particulière. La vie semble suspendue à un fil, l'inquiétude est toujours tapie dans l'ombre. Chaque instant de bonheur est fragile et menacé. C'est un amour fait d'alarmes continuelles au-dessus duquel plane sans cesse une ombre funeste.

\section{Sociologie de la vieillesse}

Cet amour que nous venons d'évoquer est aussi un amour exceptionnel car bien des œuvres de G. Wohmann viennent confirmer une réalité sociologique tout autre, la solitude des personnes âgées. Disons d'emblée que la lecture de G. Wohmann ne saurait en rien remplacer celle d'un ouvrage de sociologie consacré à la vieillesse, car l'œuvre littéraire ne prétend en rien à l'exhaustivité. Comme tout écrivain, G. Wohmann ne retient de la réalité que ce qui stimule sa créativité. En l'occurrence, c'est de ce qu'elle connaît le mieux qu'elle parle le plus volontiers. Ses personnages sont psychanalystes, universitaires, compositeurs, romanciers ; ils appartiennent - comme leur auteur - à la bourgeoisie intellectuelle. On chercherait donc vainement des thèmes comme la cassure de la retraite chez les ouvriers, le coût social de la vieillesse, les difficultés financières et tout ce qui fait de l'âge une épreuve dans les classes moins privilégiées. Malgré cet éclairage partiel sur la vieillesse, l'œuvre rejoint fréquemment les observations des sociologues, à commencer par la «seniorisation » de la société pour reprendre le concept de Serge Guérin ${ }^{13}$. L'œuvre littéraire illustre ici le vieillissement de la population allemande, corroborée par les tableaux statistiques fournis par Frank Schirrmacher dans Das Methusalem-Komplott ${ }^{14}$. En Allemagne, le pourcentage de personnes âgées de plus de 65 ans était de 14\% en 1990, il sera de 26\% en 2030. En 1990, il y avait presque trois millions d'Allemands de plus de 80 ans, en 2050 ils seront plus de 8 millions. Seuls le Japon et l'Italie atteignent des scores plus alarmants ${ }^{15}$. Cette vieillesse est éminemment féminine et l'œuvre de G. Wohmann en est le reflet. On croise bien le pasteur Bonnet, soixante-dix huit ans, dans la nouvelle Pfarrer Bonnets Start in den Tag (2008), mais le plus souvent les personnages âgés sont des femmes. Elles règnent sans partage dans le roman Bitte nicht sterben, dans la nouvelle Bald liegt Frankfurt am Meer (1991) et sont majoritaires dans le recueil Der kürzeste Tag des Jahres (1983).Cela confirme encore les chiffres fournis par F. Schirrmacher : dans la tranche de 90 à 94 ans, les femmes, en Allemagne, sont trois fois plus nombreuses que les hommes en l'an $2000^{16}$. À cela G. Wohmann n'avance pas d'explication mais on se reportera avec profit aux pistes à la fois psychologiques et physiologiques fournies par Betty Friedan au chapitre "Pourquoi les femmes vivent-

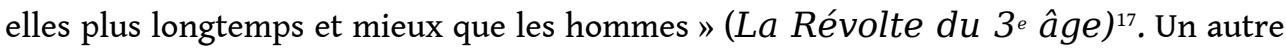
point de convergence entre sociologie et œuvre littéraire est, comme nous l'avons brièvement cité, le constat de la solitude chez les personnes âgées. C'est un thème auquel G. Wohmann a consacré de nombreuses nouvelles et un recueil entier intitulé Einsamkeit (1982). Ce recueil s'ouvre sur l'histoire d'une veuve qui s'ennuie et n'a plus avec ses enfants que des contacts épistolaires lors desquels ces derniers racontent, comme un alibi, leur quotidien accaparant. La vieille mère en vient à essayer de tomber malade pour que sa douleur se matérialise enfin. Sinon, «bien des jours demeuraient comme dépourvus de signification. On en était alors réduit à feuilleter son carnet d'adresses et de numéros de téléphone, juste pour trouver quelque part où se mettre à 
l'abri $»^{18}$. Plus pathétique encore, dans la nouvelle Der beste Freund ist in dem Himmel $^{19}$, l'héroïne confie que la seule créature à laquelle elle puisse se livrer en toute confiance, c'est Osmin..., son oiseau en cage. La monstruosité des enfants à l'égard des personnes âgées est un thème que G. Wohmann affectionne. On le retrouve dans la nouvelle Hoffentlich haben sie gutes Wetter ${ }^{20}$ (1995). L'héroïne, Mme Biederstein, a une aide à domicile qui l'incite à lui faire don de ses objets de valeur, sous prétexte que faire des cadeaux aide à rester jeune. La fille de Mme Biederstein est invitée dans le Michigan, le fils - pasteur de son état - se contente d'envoyer ses homélies à sa mère. Chacun a un bon prétexte pour manquer le quatrevingt-dixième anniversaire de la vieille dame ! Elle le passera seule. Certes, nombreuses sont les circonstances qui expliquent la solitude des personnes âgées et G. Wohmann n'en fait pas mystère - perte du conjoint, désintégration des liens familiaux, mort des amis, éloignement géographique - mais ce que G. Wohmann ne pardonne pas, c'est le cynisme de ces Gutmenschen, bobos à l'allemande, toujours prêts à militer pour la terre entière mais aveugles à la détresse la plus immédiate. Non seulement ils manquent à tous leurs devoirs filiaux, mais ils sollicitent encore l'approbation de leurs parents, comme cette fille qui dans Die Macht des Schicksals ${ }^{21}(1995)$ ne viendra pas pour Noël car elle a un billet pour l'opéra. Sa conscience est sans tache car elle est convaincue que cela fera plaisir à sa mère, qui n'aimerait sans doute pas qu'on la voie, plus guère solide sur ses jambes. G. Wohmann qui, jusqu'au bout, a rendu visite à sa mère et à ses tantes âgées est sans pitié pour ces monstres d'égoïsme. Sa représentation de la solitude dans la vieillesse apporte un bémol à des études un peu optimistes comme celle de Philippe Pitaud qui dans Solitude et isolement des personnes âgées (2004) note : «L'entraide au sein de la parenté, non seulement n'a jamais cessé d'être mais a perduré, avec une importance et une variété notables tandis que la famille connaissait des mutations profondes $»^{22}$. Gabriele Wohmann semble plutôt donner raison à Éric Cobast qui affirme que $11 \%$ des plus de 80 ans [en France] n'ont jamais aucune relation avec l'extérieur, y compris leur famille ${ }^{23}$. On se souvient aussi de Mauriac déclarant en 1965: "Un vieil homme est toujours Robinson ${ }^{24}$ ou de Jouhandeau pour qui « très peu de gens, même ceux qui les aiment, se plaisent avec les vieillards $»^{25}$. Cela ne signifie par pour autant que, chez G. Wohmann, les vieux sont prêts à tout pour " tisser du lien ", comme on dit aujourd'hui. On observe plutôt ce que les sociologues nomment une stratégie de distinction ${ }^{26}$, en d'autres termes: ce n'est pas parce que je suis vieux que je suis tenu de fréquenter des vieux pour m'arracher à la solitude. Les personnages de G. Wohmann étant depuis toujours des égocentriques et des individualistes, on ne les retrouve ni dans des clubs du troisième âge, ni dans des voyages organisés ni encore attablés autour d'une partie de bridge. Mais cette solitude, tantôt choisie, tantôt subie, laisse des séquelles. On est bien loin du prétendu «enrichissement » de la vieillesse, on est ici plutôt dans le registre du dépouillement et de l'appauvrissement. Malgré de notables convergences entre G. Wohmann et les travaux des sociologues - quand ces derniers ne versent pas dans un optimisme béat -, il est un point de divergence qui mérite d'être souligné et porte sur la place de la sexualité des personnes âgées. Betty Friedan dans $L e 3^{e}$ âge ou, plus récemment, Hermann Berberich et Elmar Brähler dans Sexualität und Partnerschaft in der zweiten Lebenshälfte (2001) ont clamé haut et fort que la vieillesse était un nouvel âge d'or pour la sexualité. Certes, quelques films comme Wolke 9 (Septième ciel) ${ }^{27}$, sorti en 2008, et certains écrivains masculins abondent en ce sens. Songeons aux romans de John Updike ou de Philipp Roth comme La tache 
(2000) dans lequel, armé de Viagra, un universitaire vieillissant fait le bonheur d'une femme de ménage de trente-quatre ans sa cadette. Il y a bien aussi le roman de Martin Walser, Der Lebenslauf der Liebe (2000), dans lequel une veuve trouve le plaisir dans la masturbation et noue une relation avec un jeune Turc. Cette représentation tient-elle au fait que ces écrivains sont des hommes? Monika Maron, pour sa part, place dans Endmoränen (2002)et Ach, Glück (2007) la vieillesse sous le signe du détachement de la chair. Dans Ach, Glück, l'héroïne quitte une relation de longue date pour se réfugier au Mexique et non auprès d'un amant. Cela fait dire au critique Ulrich Greiner dans Die Zeit : « Les femmes d'un certain âge semblent trouver qu'il y a plus important que le sexe $»^{28}$. Chez Gabriele Wohmann, la situation est plus radicale encore. La sexualité des personnes âgées, hommes et femmes, est absente. Dans le reste de l'œuvre, les héros plus jeunes ne sont déjà guère actifs. L'adultère est toujours dépeint comme décevant, le rapport sexuel comme un échange de substances visqueuses. Les personnes âgées sont, elles, tout simplement asexuées. Leur désir - s'il existe - n'est jamais évoqué si l'on excepte le personnage de Claire, la vieille dame de Bald liegt Frankfurt am Meer, qui confesse des pensées impures lors de la lecture d'un article sur les dessous masculins... Il serait absurde de voir ici le rapport de la population âgée en général à la sexualité. Nous sommes bel et bien en présence des névroses qu'un écrivain a léguées à ses personnages. Mais une nuance s'impose et elle est de taille: si la chair est absente, le désir de séduire, lui, subsiste. Ce narcissisme sénile que Simone de Beauvoir prêtait, non sans méchanceté, à Paul Léautaud nous invite à explorer plus avant le psychisme des personnages âgés peuplant l'œuvre de Gabriele Wohmann car la vieillesse n'est pas qu'une question de physiologie, c'est aussi une affaire de psychologie.

\section{Psychologie de la vieillesse}

Un des aspects de la psychologie de la vieillesse abondamment exploité par la littérature est le désir de séduction tourné vers la jeunesse, désir qui défie l'usure du corps et le qu'en dira-t-on. C'est le thème du vieux barbon qui veut plaire et de la coquette qui refuse de se voir dans un miroir. Les écrivains ont rarement été tendres, surtout avec les femmes. Erasme raille ainsi ces vieilles «qui séduisent à prix d'or quelque jeune faon, étalent des mamelles flasques et flétries, sollicitent d'une plainte chevrotante un désir qui languit, veulent boire, danser parmi les jeunes filles » (Éloge de la folie XXXII). G. Wohmann n'a pas ce ton insultant, elle se contente de sourire, mettant en scène des femmes qui, malgré la maturité, paraissent démunies devant l'illusion amoureuse, tiraillées entre désir de séduire et éclairs de lucidité cruelle. Dans la nouvelle Verschluck dich nicht (1992), Aurelia, une coquette plus toute jeune, est persuadée qu'un jeune homme la courtise. La chute est rude lorsqu'elle s'entend dire que ce qu'il apprécie chez elle, c'est... l'expérience de la vie des femmes de sa génération. Simone de Beauvoir parle de ces «femmes qui ont tout misé sur leur féminité et pour qui l'âge est une radicale disqualification. Par leurs toilettes, leur maquillage, leurs mimiques, elles cherchent à abuser autrui, mais surtout à se convaincre hystériquement qu'elles échappent à la loi commune ${ }^{29}$. Ce qu'oublient les héroïnes wohmanniennes, c'est que « la gérontophilie n'existe pas chez les jeunes gens, sauf dans des cas pathologiques, ils recherchent souvent dans leur partenaire sexuelle la mère mais non la grand-mère $»^{30}$. D'où chez les séductrices âgées une frustration affective qui est loin d'être l'unique affront réservé par la vieillesse au psychisme. 
Curieusement, longtemps les psychanalystes n'ont pas été plus tendres que les écrivains dans leur description de la personne âgée. Ferenczi (1873-1933) note: «Les gens âgés redeviennent comme des enfants narcissiques, perdent beaucoup de leurs intérêts familiaux et sociaux, une grande partie de leur capacité leur fait défaut, ils deviennent cyniques, méchants et avares ${ }^{31}$. À l'époque, Freud considérait que la cure psychanalytique ne pouvait plus rien au-delà de cinquante ans. On n'oserait plus proclamer de telles choses aujourd'hui mais psychologues et psychanalystes s'accordent à dire qu'il existe bel et bien une psychologie et des pathologies psychiques $\mathrm{du}$ vieillissement qui fournissent la matière de multiples ouvrages et dont Thomas Wallenhorst a dressé la synthèse dans La Vieillesse ${ }^{32}(2010)$. Le moment est venu d'examiner plus en détail ce que l'œuvre de G. Wohmann laisse à voir de cette psychologie de l'âge et du grand âge au-delà du démon de midi. L'un des aspects les plus marquants de la vieillesse qui s'installe et progresse est ce que les gérontologues nomment la déprise ${ }^{33}$. Au fil des années, peu à peu, les activités s'amenuisent, la curiosité intellectuelle s'émousse. On se détache de ce que l'on a aimé ou pratiqué. On ménage ses forces, on s'en tient à l'essentiel. Dans la nouvelle Pfarrer Bonnets Start in den Tag, le septuagénaire constate que ses besoins se sont peu à peu réduits à leur plus simple expression, qu'il n'a plus la patience de lire, qu'il ne prête plus attention aux tableaux dans son appartement et qu'il ne songe plus guère qu'à boire et manger. Si sa belle-fille ne jouait pas de flûte à bec, il vivrait même sans musique. Cela ne vaut pas pour tous les personnages mais un roman du grand âge comme Bitte nicht sterben est un authentique récit de déprise. On songe à la chanson de Jacques Brel, Les Vieux (1963):

Les vieux ne rêvent plus, leurs livres s'ensommeillent, leurs pianos sont fermés Les vieux ne bougent plus, leurs gestes ont trop de rides

Leur monde est trop petit, du lit à la fenêtre puis du lit au fauteuil et puis du lit au lit.

Ce ralentissement progressif va de pair avec un nouveau rapport au temps. Pour beaucoup, vivre se limite désormais à endurer la durée. Le temps, vide de contenu, stagne ou se répète indéfiniment jusqu'à abolir toute notion de temporalité conçue comme dynamique. C'est le terreau propice à l'éclosion de l'ennui. Dans Der Klügere gibt nach (1992), Mathilde, qui a le malheur d'être matinale, souffre du vide, sitôt levée. Beaucoup de personnages comme les vieilles femmes de Bitte nicht sterben cherchent à se soustraire à l'ennui en reprenant le matin un cachet de Rohypnol pour prolonger la nuit. On n'est jamais très loin de la dépressivité. Les personnages sentent que "vieillir, c'est en quelque sorte toucher à une forme d'impossibilité. La vieillesse n'a rien d'un mal guérissable $»^{34}$. Puisque la partie est perdue, comment mettre "à profit» le temps qui reste? La protagoniste de Der siebzigste Geburtstag envie aux autres leur "provision de sens $»^{35}$. Il lui arrive de connaître quelques embellies: «Ma foi, il m'arrive d'éprouver un sentiment de CARPE DIEM, et c'est pour ces beaux jours que je vis $»^{36}$ mais ces éclaircies sont de courte durée. Quelques lignes plus loin, la même femme décrit sa vie comme un "demi-malheur perpétuel $»^{37}$ et s'apprête à déclarer à son amie que la vie est ennuyeuse. L'envie qu'elle éprouve à l'égard des autres ne se limite pas à leur provision de sens. C'est plus généralement une jalousie à l'endroit de ceux qui ont la vie devant eux et non derrière eux. Cette femme va jusqu'à rompre avec son amie Verena : «Chère Verena, mon monde s'achève, mais vous, toi et ta famille, avez encore le vent en poupe. Cela commence à m'ennuyer, je te le dis franchement $»^{38}$. Parfois, l'aigreur confine au sadisme. Pour se venger de leurs enfants, 
trop jeunes, trop bien portants, trop occupés, certains personnages se font un plaisir d'être déplaisants et d'inquiéter leur progéniture, comme Mme Kaulbach qui, dans la nouvelle Infektionskrankheiten (1984) prétend ne plus avoir d'appétit. En faisant faux bond au médecin et à sa femme de ménage, elle retrouve le malin plaisir qu'elle avait, enfant, à sécher les cours. On songe ici à l'inoubliable Tatie Danièle d'Étienne Chatilliez. Parmi les autres traits récurrents de la psychologie des personnes âgées wohmanniennes, on notera encore un manque de souplesse qui fait que les petites anicroches tournent vite à l'incident diplomatique. La patience se perd au fil du temps. On observe également une ritualisation du quotidien. La vie des héroïnes de Bitte nicht sterben est réglée comme du papier à musique. Elles découpent des histoires drôles dans le journal, sélectionnent des articles à faire lire aux enfants lorsqu'ils viendront, nourrissent les oiseaux et le chien, passent quelques coups de téléphone et s'installent devant la télévision selon un rituel immuable. C'est ce qu'elles nomment la préservation du statu quo, c'est le moyen de conjurer l'angoisse, de ne pas penser à la catastrophe majeure - la mort - ni aux catastrophes mineures - les maux en tous genres qui les accablent. Mais quels que soient les efforts qu'elles déploient, toujours la mort est tapie dans l'ombre. Ce spectre suscite de nombreuses frayeurs, à commencer par l'hypocondrie. Die Bagatelle-Balance (1984) présente un cas intéressant d'hypocondrie par procuration. Silja Knecht, l'héroïne, soupçonne chez son mari d'horrifiques pathologies. Un kyste sur la langue devient aussitôt un cancer. À noter toutefois que l'hypocondrie chez G. Wohmann n'est pas spécifique à la vieillesse. Elle est le fruit de l'introspection maladive des personnages, d'une inactivité fâcheuse et d'une imagination fertile. Sans penser nécessairement au pire, une question qui taraude les personnages est celle du temps qui leur reste: "me réveillerai-je demain?", leur chuchote une voix à l'oreille? Au-delà de la peur face à leur propre condition de mortels, les personnages sont tétanisés à l'idée, exprimée déjà par Juvénal, que « vieillir, c'est voir mourir ceux qui nous sont chers, c'est être condamné au deuil et à la tristesse $"^{39}$. Cette hantise des rangs qui s'éclaircissent est accentuée chez G. Wohmann par une hyperesthésie affective qui fait qu'aimer, c'est toujours aimer trop. À partir d'un certain âge, le défi à relever consiste à vivre en intégrant la mort de l'autre. Ce thème a fourni à l'écrivain la matière de nombreux romans dont le récit autobiographique Abschied von der Schwester (2001). Les personnages s'avouent souvent démunis : «Parce que deux de mes frères et sœurs sont plus âgés que moi, je suis poursuivie par le souci de leur santé ! Ah, vivre sans inquiétude! Je ne sais pas comment font les gens ${ }^{40}$, soupire l'héroïne de Der siebzigste Geburtstag. Chez G. Wohmann, les liens qui rattachent les personnages aux êtres aimés sont comme des fers qui arrachent les chairs lorsque l'un disparaît. S'ajoute l'angoisse, si l'on meurt en premier, de laisser l'autre seul et abandonné. Dans Erzählen Sie mir was vom Jenseits, la romancière confesse sa fascination pour le poème de Reiner Kunze, Bittgedanke, Dir zu Füßen :

Stirb früher als ich, um ein weniges

früher

Damit nicht du

den Weg zum Haus

allein zurückgehen musst ${ }^{41}$.

On le voit, l'œuvre de Gabriele Wohmann donne raison à Ulrich Greiner qui affirme dans "Länger, nicht leichter "42 (Die Zeit) que le progrès a prolongé la vieillesse mais qu'il ne l'a pas rendue plus facile. À noter que le tableau que G. Wohmann dresse 
de la psychologie de la vieillesse n'est pas exhaustif. On n'y trouve ni la paranoïa du grand âge, ni l'avarice pathologique ni encore la démence sénile mais le tableau est suffisamment riche pour illustrer ce passage d'Aristote dans La Politique : «L'esprit, tout comme le corps, est soumis à la vieillesse. C'est précisément en vertu de l'union de l'âme et du corps que la décrépitude de l'un atteint immanquablement l'autre $»^{43}$.

\section{Vieillesse et métaphysique}

Étonnamment, beaucoup d'ouvrages consacrés à la vieillesse passent sous silence la question de la mort et de l'après. Dans son livre intitulé La vieillesse, Éric Cobast ne consacre ainsi que deux pages à la mort et son chapitre "Bien vieillir pour bien mourir » est consacré à... l'espérance de vie. L'auteur note: «ce qu'une étude sur la vieillesse pourrait occulter, c'est notre rapport à la mort $»^{44}$. Pour Frank Schirrmacher dans Das Methusalem-Komplott, cette position n'est pas acceptable. Selon lui, la vieillesse rend incontournable le questionnement métaphysique. Il écrit: "Il est évident qu'avec un nombre croissant de personnes âgées et dans une société qui considère le vieillissement comme dramatique les «questions ultimes " se posent avec une vigueur toute nouvelle: d'où venons-nous? Où allons-nous? Et pourquoi ? »5. L'œuvre de G. Wohmann lui donne raison. N'en déplaise à Bossuet selon qui « c'est une étrange faiblesse de l'esprit humain que jamais la mort ne lui soit présente, quoiqu'elle se mette en vue de tous côtés, et en mille formes diverses $»^{46}$, les personnages wohmanniens, et notamment les plus âgés, vivent avec une préoccupation constante de la mort et de l'après. Cette inquiétude métaphysique se manifeste nettement à partir du roman Schönes Gehege (1975). Le héros, le romancier Robert Plath, vit avec angoisse les dernières semaines qui précèdent la mort de son père âgé. C'est pour le romancier l'occasion de méditer sur ce qui attend le mourant. Sera-t-il uniquement la proie des vers ? Est-il possible que de cette vie d'homme il ne reste plus rien ? Soudain, Robert Plath se révolte. Il refuse de se plier aux arguments des rationalistes pour qui la vie n'est qu'un processus physico-chimique et qui s'acharnent à démontrer qu'il n'y a nulle part ailleurs dans l'univers de traces de vie. À la suite de Kierkegaard, Robert Plath jette par-dessus bord les "vérités » scientifiques et décide de croire que la mort n'est pas l'écœurante conclusion de toute chose mais le début de ce que l'esprit humain ne parvient à concevoir. Robert Plath n'attend pas que Dieu se manifeste à lui, il décide de croire en Lui et considère soudain la mort sous un angle différent: " Si seulement la mort était bien comprise comme le passage à l'être véritable - quoiqu'il faille hélas bien l'admettre : être inconnu - alors seulement on pourrait vivre et mourir raisonnablement. Vivre avec la mort $»^{47}$. À cela, Robert Plath décide de s'entraîner chaque jour, aidé par Luther, Kierkegaard et Karl Barth. On reconnaît ici l'héritage de Gabriele Wohmann, élevée par un père pasteur dans la foi luthérienne, père aimé, adulé, dont la perte en 1974 constitua un séisme psychologique. Le roman Schönes Gehege (1975) se voulait l'amorce d'une guérison. Avec ce roman d'adhésion à la foi, on eût pu croire définitivement balayée toute inquiétude métaphysique. Pourtant il n'en fut rien. Les rechutes abondèrent. L'ennui existentiel guettait toujours, la tentation du suicide était toujours présente. En 1992, G. Wohmann met en scène dans la nouvelle Grafschaft une femme qui, après avoir refermé les écrits d'un grand théologien, part se faire volontairement piétiner par des chevaux au galop pour échapper à l'angoisse et à l'ennui. Dans Bitte nicht sterben, les vieilles dames, malgré leur foi, n'excluent pas non plus le suicide si Alzheimer ou la vieillesse devaient 
les réduire à de cruelles extrémités. Le mari de la narratrice s’en émeut : «Que disent donc tes prophètes à propos du suicide? Tous les Karl Barth, les apôtres et les Kierkegaard ? $»^{48}$. - «Dieu n'a rien contre $»^{49}$, lui répond sa femme. On comprend mieux dès lors que $\mathrm{G}$. Wohmann, qui ne fait pas mystère de sa foi, refuse l'étiquette d'écrivain chrétien. Elle ne veut pas mettre en scène « des êtres qui ont déjà trouvé leur but, dont l'assurance dans la foi est entière, qui chantent joyeusement des cantiques, font leur prière et terminent leurs journée bien contents $»^{50}$. Ce qui stimule sa créativité, ce sont les êtres déchirés. Avec une grande humanité, G. Wohmann met donc en scène des chrétiens qui veulent croire en Dieu mais qui tremblent devant la mort et c'est cette tension métaphysique qui sous-tend la représentation de la vieillesse dans l'œuvre. "Qui, lorsque je prends chacun de nous individuellement, apprécie vraiment sa vie? Qui n'arrive pas, au bout du compte, qu'à quelques moments par-ci, par-là qu'il apprécie vraiment? $»^{51}$ demande la narratrice de Bitte nicht sterben à ses parentes âgées qui, toutes, ont la foi. Avant d'ajouter que pourtant, "mourir, ne plus être là, sérieusement, aucun de nous qui sommes assis là ne le souhaite. Nous avons tous mortellement peur de la mort $»^{52}$. Gabriele Wohmann ne se moque pas. Elle sait que cette peur viscérale de l'inconnu qu'est la mort est par trop humaine. On a beau chanter cent fois par jour avec confiance Komm o komm du Todesstunde, il suffit d'un élancement soudain dans la région du cœur pour que la panique l'emporte. Même le pasteur Bonnet (Pfarrer Bonnets Start in den Tag), malgré ses soixante-dixhuit ans, avoue n'être pas prêt à mourir quoiqu'il prétende s'y exercer constamment. Il a beau être pasteur, il n'en est pas moins homme. Et sa peur de l'inconnu n'a rien de blâmable. Pour le théologien avec lequel Gabriele Wohmann s'entretient dans Erzählen Sie mir was vom Jenseits, "un chrétien ne serait pas un homme, si cette peur ne le saisissait pas $»^{53}$. Les chrétiens wohmanniens sont donc bel et bien humains, avec les faiblesses que cela implique.

Pour conjurer leur angoisse de la mort, ces hommes et ces femmes cherchent désespérément des réponses et se tournent naturellement vers le clergé puisque de tout temps sa mission a consisté à réconcilier et familiariser les croyants avec la mort. Pourtant, si l'on en croit Gabriele Wohmann, ces pauvres chrétiens sont bien mal inspirés car cela fait longtemps que le clergé a cessé de parler de la mort et de l'au-delà. C'est l'occasion pour l'écrivain de faire de ce clergé qui abandonne les plus âgés à leur peur de l'inconnu un portrait au vitriol. Dans Bitte nicht sterben, le pasteur rend visite à ses ouailles comme il est d'usage lorsqu'elles fêtent leur quatre-vingtième anniversaire. Marie-Rosa, l'une des vieilles dames, qui sait que ses années sont désormais comptées, espère secrètement que l'homme d'Église va lui parler de ce qui attend le chrétien. Au lieu de cela, pour être dans l'air du temps et ne pas effrayer ses fidèles, le pasteur ne dit pas un mot de la mort et, comme s'il ne croyait pas à l'au-delà, invite les vieilles dames à chercher le bonheur ici-bas en dépeignant sous des couleurs radieuses les prétendus plaisirs de la vie et les joies terrestres: "C'est ici et aujourd'hui, chère Mme Lietzmann, ici sur terre, que nous devrions, chacun en soi et autour de soi, chercher le paradis $»^{54}$. Il s'enfonce encore un peu plus en vantant la sagesse du bouddhisme. Pour Marie-Rosa l'affaire est entendue, ce n'est pas un pasteur, c'est un athée. Cette charge de G. Wohmann, fille de pasteur, contre la trahison du clergé n'est pas isolée. On la retrouve ailleurs, ainsi dans Die Vermischung der Lehren ${ }^{55}$ (1988). Malgré le côté caricatural, l'écrivain met en exergue une réalité, l'incapacité croissante du clergé à parler de la mort, incapacité qui laisse sur leur faim des personnes âgées en quête de consolations. À en croire le bénédictin Anselm Grün 
dans Was kommt nach dem Tod ${ }^{56}$ (Se réconcilier avec la mort) (2008), chez les prêtres catholiques, cette tendance se situe dans le droit fil du concile de Vatican II à la suite duquel l'Église a supprimé de sa liturgie les textes susceptibles d'effrayer, ainsi le passage Dies irae, dies illa dans le Requiem. Dans un même mouvement, l'enfer a disparu des sermons et de la théologie contemporaine. À la décharge des pasteurs et des prêtres, il convient de souligner que parler du ciel n'est pas chose aisée. Anselm Grün en convient :

Contrairement aux musulmans, les chrétiens ont souvent du mal à décrire le ciel qui les attend. La théologie l'a souvent dépeint de manière très abstraite comme un lieu où l'homme ne forme qu'un avec Dieu. Comment pouvons-nous, chrétiens, nous imaginer le ciel? Quelles que soient les images que nous en concevons, elles demeurent toujours en-deçà de ce qui nous y attend. Paul ne dit-il pas qu'aucun œil n'a vu, qu'aucune oreille n'a entendu ce que Dieu a préparé pour nous ? ${ }^{57}$

Mais, et c'est ce que suggère G. Wohmann, s'ils s'en donnaient la peine, prêtres et pasteurs trouveraient en abondance dans la Bible de quoi apaiser les angoisses des personnes âgées effrayées par l'inconnu. Dans l'évangile selon saint Jean 14/1-3, Jésus promet de préparer à chacun une maison dans la demeure de son père. Paul exhorte les chrétiens à ne pas céder à l'abattement: «Ne soyez pas dans la tristesse comme les autres qui n'ont pas d'espérance: nous croyons que Jésus est mort et qu'il est ressuscité, de même aussi ceux qui sont morts, Dieu les accueillera par Jésus et avec lui » (1 Thessaloniciens 4,13-14). C'est dans cet esprit qu'Anselm Grün a rédigé Was kommt nach dem Tod ? : "Je voudrais mettre en lumière à travers les images que nous offre la Bible et la liturgie ce que la mort nous réserve et ce que nous sommes, chrétiens, en droit d'espérer $\aleph^{58}$. Il sait que rien ne pourra supprimer la peur de mourir car elle appartient intrinsèquement à la nature humaine mais il croit possible d'amener les chrétiens à ce que Gabriele Wohmann nomme "eine getroste Angst " ${ }^{59}$, une peur à laquelle se mêle la confiance, peur de l'inconnu mêlée à l'espoir de ne pas être abandonné. Anselm Grün décrit cette getroste Angst ainsi : « les images de la Bible agissent à l'encontre de notre peur; elles transforment celle-ci en espoir et sérénité ${ }^{60}$. Malheureusement, les pasteurs wohmanniens ne sont pas Anselm Grün. Ils laissent leurs ouailles inconsolées, les obligeant à chercher ailleurs les consolations qu'on leur refuse. C'est dans la musique de Jean-Sébastien Bach que les personnages wohmanniens s'approchent de cette getroste Angst. Les héroïnes de Bitte nicht sterben ainsi que Felicitas, la femme âgée et malade de Bald liegt Frankfurt am Meer,fredonnent des cantates. Mises en musique, l'angoisse, la mort perdent de leur effroi. Dans L'Esthétique de Jean-Sébastien Bach, André Pirro note: "Chaque fois que le texte contient une allusion à la mort, les motifs de l'assoupissement bercent la composition. Que dans le cours d'un air ou d'un récitatif la mort soit évoquée, les calmes séries de sons apparaissent. Des pièces entières sont rythmées par le murmure des instruments graves qui annoncent le sommeil : Endormez-vous, ô mes yeux épuisés » ${ }^{61}$. À cela, dans Jean-Sébastien Bach, Roland de Condé ajoute: «La Todessehnsucht, la nostalgie joyeuse de la mort, l'intense désir du repos éternel, voila bien le témoignage le plus fort et le plus constant de la foi de Jean-Sébastien Bach ${ }^{62}$. C'est de ces témoignages que les personnages de G. Wohmann ont soif. Lorsque les vieilles femmes de Bitte nicht sterben écoutent ou fredonnent Bach, on songe à cette expérience musicale décrite par Cioran, lequel recommande la musique de Mozart et de Bach comme remède au désespoir : «autrefois inconsolable, voilà que vous poussent des ailes qui vous élancent dans un vol serein, accompagné de sourires discrets et voilés, dans une éternité de charme évanescent et de transparences douces 
et caressantes ${ }^{63}$. Mais que l'on ne s'y méprenne pas, G. Wohmann n'a jamais eu pour ambition de rédiger des ouvrages de développement personnel promettant une vieillesse apaisée grâce à la musique. Rien n'est plus éloigné d'elle que ces balivernes sur l'art de "positiver" la vieillesse, laquelle demeure à ses yeux bel et bien un outrage. Si l'espoir s'insinue pourtant à la lecture des romans et nouvelles que G. Wohmann a consacrés à la vieillesse, c'est parce que l'écrivain refuse de céder au désespérant matérialisme ambiant et qu'elle n'envisage jamais le sujet sans se poser la question des fins ultimes. À mille lieues de certitudes édifiantes, l'auteur de Erzählen Sie mir was vom Jenseits suggère à l'oreille du lecteur, qu'elle imagine, comme elle, en quête de consolations : «Et si l'on parlait un peu de Dieu?». En effet, si l'on en croit sa nouvelle Infektionskrankheiten, « la vérité est que seule la foi en Dieu peut rendre la mort attrayante $\aleph^{64}$. On comprend mieux dès lors que pour le théologien Georg Magirius l'œuvre de Gabriele Wohmann soit à apprécier comme « une bouffée de bonheur pour ceux qui ne se satisfont pas de l'ici et maintenant $»^{65}$.

\section{NOTES}

1. Catherine Bergeret-Amselek, La Vie à l'épreuve du temps, Paris, Desclée de Brouwer, 2009, p. 67.

2. Éric Cobast, La Vieillesse, Paris, Ellipses, 2010, p. 14.

3. Ibid., p. 14.

4. Simone de Beauvoir, La Vieillesse, Paris, Gallimard, 1970.

5. Dans les notes qui suivent, G. W. désignera Gabriele Wohmann.

«Puh, ich seh ja wie eine alte Frau aus, hatte sie innerlich gestöhnt, als sie sich, bei schlechter Laune in einem Spiegel ertappt hatte. Ihr war erst später aufgefallen, dass sie ja eine alte Frau war, aber da, weil sie lachen musste, hatte sie schon nicht mehr diesen Eindruck von sich als alter Frau gehabt ». G.W., Der beste Freund ist in dem Himmel in Der kürzeste Tag des Jahres, DarmstadtNeuwied, Luchterhand, 1983, p. 6.

6. «Das Alter? Früher habe ich sofort Schopenhauerprosa verstanden. Früher kam ich, ohne zu keuchen, einen Abhang rauf. Früher brachte mich nicht jeder Luftzug aus der Fassung, und mit zerzaustem Haar sah ich gut aus. Früher habe ich behalten, was ich gelesen habe. Früher brauchte ich keinen Betablocker und keine Brille. Früher habe ich in aller Ruhe genießerisch an den Tod gedacht und überhaupt niemals an meine Verdauung ». G.W., Verschluck dich nicht in , Das Salz, bitte!", Munich, Piper, 1992, p. 60.

7. Béatrice Jongy, «Si je suis vivant » : vieillir dans les journaux et autoportraits du $\mathrm{xx}^{\mathrm{e}}$ siècle » in D'âge en âge, Paris, L'Harmattan, 2008, p. 161 sq.

8. Claudine Chevallier, «La vieillesse en littérature », in La Vieillesse. Concours IEP, Paris, Ellipses, 2010, p. 148 sq.

9. Éric Cobast, op. cit., p. 15.

10. «[...] all die munteren Senioren mit ihren Supersparpreisreisen, dem Überwintern auf Mallorca, [...] alles Heuchelei. Von den Kranken, Hilflosen, den Demenz-Greisen in Zwei- und Dreibettzimmern in Pflegeheimen wird weniger gern geredet ", Bitte nicht sterben, Munich, Piper, 1993, p. 81 .

11. " die fast viktorianische körperfeindliche Einstellung », Klaus Wellner, Leiden an der Familie, Stuttgart, Ernst Klett Verlag, p. 196. 
12. «Wir haben gegen eine Art Prüderie angesichts der Tatsachen zu kämpfen. Vor der Zukunft strecken wir die Waffen. Wir fühlen uns unfair, taktlos, wenn wir die Hilflosigkeit des Altseins auch nur andeuten, wir halten uns an ein altmodisches Davon-spricht-man-nicht und lassen uns in unseren Spielton zurückfallen ", Bitte nicht sterben, p. 297.

13. Cf. Serge Guérin, La Société des seniors, Michalon, 2009.

14. Frank Schirrmacher, Das Methusalem-Komplott, Munich, Karl Blessing Verlag, 2004.

15. Cf. Nations-Unies, Population Division, 1999, cité par F. Schirrmacher, op. cit., p. 44.

16. Berlin-Institut für Weltbevölkerung und globale Entwicklung, cité par F. Schirrmacher, op. cit., p. 47.

17. Betty Friedan affirme : «Au cours des années pendant lesquelles elles ont embrassé la cause du féminisme, les femmes ont, semble-t-il, cessé de souffrir des troubles mentaux et des détresses physiques qui diminuaient leur force [...]. Les femmes sont plus sensibles aux moindres modifications de leur corps ou de leur santé, plus enclines à signaler une maladie, à se soigner ", La Révolte du $3^{e}$ âge, p. 106. G. Wohmann émettrait toutefois certaines réserves. Son bilan du féminisme est moins élogieux et chez elle, l'hypocondrie se répartit équitablement entre hommes et femmes.

18. «Manche Tage blieben wie ohne Motiv. Und dann wurde man so weit gebracht, dass man im Adressen- und Telefonnummernbüchlein herumblätterte, um sich bloß irgendwo unterzubringen », Einsamkeit, p. 9.

19. Recueil Der kürzeste Tag des Tages, Darmstadt-Neuwied, Luchterhand, 1984, p. 7 sq.

20. Recueil Die Schönste im ganzen Land, Munich, Piper, 1995.

21. Ibid.

22. Philippe Pitaud, Solitude et isolement des personnes âgées, Ramonville-St Agne, éditions érès, 2004, p. 45

23. Éric Cobast, op. cit., p. 9.

24. Cité par Bernadette Puijalon, «Paroles de solitude » in Solitude et isolement des personnes âgées, Ramonville-St Agne, éditions érès, 2004, p. 13.

25. Ibid., p. 17.

26. Ibid., p. 87.

27. Wolke 9 (Septième ciel) est un film d'Andreas Dresen qui montre sans tabou la passion physique entre Inge, sexagénaire, et Karl, un homme de 76 ans.

28. "Ältere Frauen scheinen anderes wichtiger zu finden als Sex », Ulrich Greiner, "Länger, nicht leichter », Die Zeit, 20/09/2007.

29. Simone de Beauvoir, op. cit., p. 313.

30. Ibid., p. 234.

31. Cité par C. Bergeret-Amselek, op. cit., p. 54.

32. Thomas Wallenhorst, "Psychologie et pathologies psychiques du vieillissement" in $L a$ Vieillesse, Concours commun IEP, Paris, Ellipses, 2010. Nous renvoyons aussi aux travaux de la psychanalyste C. Bergeret-Amselek, cf. supra.

33. Cf. S. Clément, M. Membrado, «Expériences du vieillir : généalogie de la notion de déprise » in Penser les vieillesses, Paris, éditions Seli Arslan, 2010, p. 117.

34. Éric Cobast, op. cit., p. 16.

35. G.W., Le Soixante-dixième anniversaire in Le Seigneur est un ami, Paris, Verdier, p. 156.

36. Ibid., p. 153.

37. Ibid., p. 156.

38. Ibid., p. 152.

39. Cf. Simone de Beauvoir, op. cit., p. 133.

40. G.W., Le soixante-dixième anniversaire in Le Seigneur est un ami, p. 151.

41. Reiner Kunze, Bittgedanke, Dir zu Füßen cité par G. Wohmann in Erzählen Sie mir was vom Jenseits, Mayence, Matthias-Grünewald-Verlag, 1994, p. 46. 
42. Ulrich Greiner, « Länger, nicht leichter », Die Zeit, 20/09/2007.

43. Cité par E. Cobast dans La Vieillesse, p. 49.

44. Ibid., p. 108.

45. « Klar, dass mit einer wachsenden Anzahl von Älteren und in einer Gesellschaft, die Altern als schlimm empfindet, die "letzten Fragen" eine ganz neue Macht entfalten : Woher kommen wir? Wohin gehen wir? Und warum?", Das Methusalem-Komplott, p. 132.

46. Bossuet, Sermon sur la mort (1662), Paris, Garnier Flammarion, 2004, p. 129.

47. «Würde doch der Tod richtig verstanden, als der Übergang zum wahren, wenn auch zugegebenermaßen leider unbekannten Sein, dann nur könnte vernünftig gelebt und gestorben werden. Mit dem Sterben gelebt werden ", Schönes Gehege, p. 210.

48. "Was sagen denn deine Propheten zum Selbstmord? All die Karl Barths und Apostel und Kierkegaards?», Bitte nicht sterben, p. 302.

49. «Gott hat nichts dagegen ", ibid.

50. «Menschen, die ihr Ziel bereits gefunden haben, die im Glauben völlig sicher sind und fröhlich Gesangbuchlieder singen, ihr Gebet sprechen und dann in Zufriedenheit ihren Tag beschließen ", Kultur-Chronik, 2/1997, Bonn/Bad Godesberg, Inter-Nationes, p. 16.

51. «Wer, wenn ich jeden von uns einzeln vornehme, hat sein Leben wirklich gern ? Wer kommt nicht nur auf einzelne Momente, die er wirklich gern hat ?», ibid., p. 298.

52. «Sterben, nicht mehr da sein, im Ernst will das keiner von uns allen, wie wir hier sitzen. Alle haben wir eine Todesangst vor dem Tod », ibid.

53. " Ein Christ wäre ein Unmensch, wenn diese Angst ihn nicht ergriffe », Erzählen Sie mir was vom Jenseits, p. 162.

54. « Hier und heute, liebe Frau Lietzmann, hier auf Erden sollten wir, jeder in sich selber und um sich herum, das Paradies suchen », Bitte nicht sterben, p. 49.

55. Nouvelle du recueil Ein russischer Sommer.

56. Anselm Grün, Was kommt nach dem Tod ?, Münsterschwarzach, Vier-Türme-Verlag, 2008. Nous citons d'après la traduction française de Caroline Jouannic : Se réconcilier avec la mort, Paris, Albin Michel, 2009.

57. Anselm Grün, Se réconcilier avec la mort, p. 92.

58. Ibid., p. 11.

59. G.W., Erzählen Sie mir was vom Jenseits, p. 162.

60. Anselm Grün, op. cit., p. 12.

61. André Pirro, L'Esthétique de Jean-Sébastien Bach, (1927), cité d'après l'éd. Minkoff, Genève, 1973, p. 453.

62. Roland de Condé, Jean-Sébastien Bach, Paris, Seuil, 1984, p. 261.

63. E.M. Cioran, Le Livre des leurres in Euvres, Paris, Gallimard, 1995, p. 138.

64. "Die Wahrheit ist, dass allein der Gottesglaube den Tod schmackhaft machen kann", Infektionskrankheiten in Der kürzeste Tag des Jahres, p. 66.

65. «Eine Glückszufuhr für jene, die mit dem Hier und Jetzt nicht zufrieden sind», Georg Magirius, préface de l'ouvrage de G. Wohmann, Sterben ist Mist, der Tod aber schön: Träume vom Himmel, Freiburg im Breisgau, Kreuz Verlag, 2011, p. 7. 


\section{RÉSUMÉS}

La romancière et nouvelliste allemande Gabriele Wohmann, née en 1932 à Darmstadt, fêtera en 2012 son quatre-vingtième anniversaire. Depuis longtemps déjà, la vieillesse et ses manifestations font partie de ses préoccupations. Au fur et à mesure que l'écrivain vieillissait, le sujet n'a fait que gagner en importance. En 1993, c'est un roman entier, largement autobiographique, que Gabriele Wohmann consacrait au grand âge, Bitte nicht sterben. C'est ainsi qu'ouvrage après ouvrage toute une galerie de personnages âgés a vu le jour, venant illustrer les connaissances physiologiques, sociologiques et psychologiques en matière de gérontologie. Toutefois, Gabriele Wohmann n'est pas une scientifique. Ce qui l'intéresse avant tout dans la vieillesse, c'est l'incontournable réflexion métaphysique sur ce qu'il est convenu d'appeler les questions ultimes, et notamment : où allons-nous ? C'est ainsi qu'il n'est pas rare que les considérations de l'écrivain recoupent celle du moine bénédictin Anselm Grün, dans son ouvrage Was kommt nach dem Tod (2008). Dans une littérature contemporaine dont Dieu est souvent absent, cela confère à la voix de Gabriele Wohmann des accents uniques et impossibles à ignorer.

Die 1932 in Darmstadt geborene Romanschriftstellerin und Kurzgeschichtenautorin Gabriele Wohmann wird 2012 achtzig Jahre alt. Schon längst aber beschäftigt sie das Alter und seine Erscheinungen. Je älter die Autorin wurde, umso gewichtiger wurde das Thema in ihrem Werk. 1993 hat Gabriele Wohmann sogar einen ganzen, weitgehend autobiographischen Roman dem hohen Alter gewidmet: Bitte nicht sterben. So entstand Buch für Buch eine Galerie von alten Menschen, die die medizinischen, soziologischen und psychologischen Erkenntnisse der Altersforschung veranschaulichen. Gabriele Wohmann ist aber keine Wissenschaftlerin. Am meisten interessiert sie am Alter die unverzichtbare metaphysische Reflexion über die sogenannten letzten Fragen, insbesondere : Wohin gehen wir ? So kommt es, dass sich ihre Betrachtungen nicht selten mit denen des Benediktinermönchs Anselm Grün in seinem Buch Was kommt nach dem Tod ? (2008) decken. In der Gegenwartsliteratur, für die Gott oft kein Thema mehr ist, verleiht dies der Stimme Gabriele Wohmanns einen unverwechselbaren und unüberhörbaren Ton.

The German novelist and short-story writer Gabriele Wohmann, born in 1932 in Darmstadt, will turn eighty in 2012. However, advancing age and its manifestations started to be a topic for her long ago. The older the author grew, the more important this subject became in her work. In 1993 Gabriele Wohmann has even dedicated an entire, to a large extent autobiographical novel, Bitte nicht sterben, to very old age. Book after book she created a gallery of old people who illustrate the medical, sociological and psychological aspects of gerontology. But Gabriele Wohmann is not a scientist. What interests her most about aging is the inevitable metaphysical reflection about the so-called "final questions", especially: Where are we going? This explains why her considerations frequently remind of the Benedictine monk Anselm Grün's book Was kommt nach dem Tod? (2008). In contemporary literature in which God often is no longer a topic, this makes Gabriele Wohmann's voice unique and impossible to ignore.

\section{INDEX}

Mots-clés : Allemagne, autobiographie, réflexion métaphysique, vieillesse 
AUTEUR

BENOÎT PIVERT

Université de Paris-Sud (Paris XI) 